\title{
Información asimétrica e inclusión financiera en Piura
}

\author{
Pablo Rijalba Palacios \\ prijalbap@unp.edu.pe \\ Facultad de Economía de la Universidad Nacional de Piura \\ Programa de Doctorado en Economía y Finanzas (PRODACEF) y \\ Programa de Doctorado en Administración de la EUPG - \\ Universidad Federico Villarreal (UNFV) \\ Piura - Lima - Perú
}

\section{RESUMEN}

Se analiza el desempeño financiero $\boldsymbol{y}$ social de las microfinanzas en Piura como respuesta al fallo de mercado: asimetrías de información. En base a aportes de la microeconomía moderna se infiere que estas se extienden a las finanzas inclusivas vía selección adversa, riesgo moral y comportamiento de rebaño (Stiglitz,2002), evidenciando débiles relaciones sociales y contractuales pese al buen desempeño de las IMF. Se aplicó metodologías cuantitativas basadas en análisis factorial y de respuestas binarias; y utilizando data del ENAHO (2019) y SBS (2019), se encuentra que, en presencia de asimetrías, no es suficiente el buen desempeño financiero de las IMF, pues estamos ante un proceso social que requiere de políticas públicas para regular su funcionamiento.

Palabras clave: Asimetría; finanzas inclusivas; desempeño financiero y social. 


\title{
Asymmetric information and financial inclusion in Piura
}

\begin{abstract}
The financial and social performance of microfinance in Piura is analyzed in response to the market failure: information asymmetries. Based on contributions from modern microeconomics, it is inferred that these extend to inclusive finance via adverse selection, moral hazard and herd behavior (Stiglitz, 2002), showing weak social and contractual relationships despite the good performance of MFIs. Quantitative methodologies based on factorial analysis and binary responses were applied; and using data from ENAHO (2019) and SBS (2019), it is found that, in the presence of asymmetries, the good financial performance of MFIs is not enough, since we are facing a social process that requires public policies to regulate its operation.
\end{abstract}

Keywords: Asymmetry; inclusive finance; financial and social performance.

Artículo recibido: 05 de Mayo 2021 Aceptado para publicación: 20 de Junio 2021 Correspondencia: prijalbap@unp.edu.pe Conflictos de Interés: Ninguna que declarar 


\section{INTRODUCCIÓN}

El propósito es reflexionar sobre la forma como se presentan las asimetrías de información en procesos de inclusión financiera para espacios locales como Piura. La motivación, va más allá del interés por reconocer las imperfecciones de los mercados financieros locales y se centra en el hecho de haber observado que el buen desempeño de las Instituciones Microfinancieras (IMF) que operan en Piura no estaría reflejando procesos financieros inclusivos fuertes y sostenibles debido a la presencia de asimetrías que no han sido suficientemente evaluadas desde los componentes que lo determinan.

A partir de Stiglitz (2002) se busca mostrar que, pese al buen desempeño de las IMF, la inclusión financiera es mucho más que un buen desempeño y sostenibilidad de las IMF, es un proceso social que requiere participación y vigilancia del estado. Se sostiene aquí que, estaríamos frente a un problema que debe ser abordado desde la política pública, pues no hay suficiente evidencia de si la inclusión financiera mejora las condiciones de vida de manera sostenida; y en gran medida, debido a que las asimetrías informativas afectan los procesos financieros inclusivos vía relaciones contractuales, selección adversa, riesgo moral y comportamiento de rebaño. Como respuesta a ello, las IMF procurarían el racionamiento de fondos y sesgan el acceso/uso de los servicios financieros. Por ello, que se hace necesario reconocer la importancia de estas asimetrías identificarlas como parte de relaciones contractuales incompletas.

En esta interacción, IMF y usuarios no poseen toda la información relevante del comportamiento de su contraparte. Ni IMF ni potenciales sujetos de crédito cuentan con suficiente información para fortalecer procesos financieros, ya sea por falta de educación financiera, por condiciones de informalidad, por insuficiente claridad en las relaciones contractuales crediticias, por condiciones crediticias excluyentes o incluso por insuficiente regulación relacionada a este sector. Es así que ninguna de las partes tienen certeza $\boldsymbol{a}$ priori, para reconocer la probabilidad de incumplimiento del crédito, así como en la detección y determinación de riesgos.

En Piura las microfinanzas se han desarrollado ampliamente, pero no se conoce aún la dimensión de su contribución a desarrollos inclusivos. No se ha estudiado de manera suficiente, si el buen desempeño de las IMF ha contribuido a mejorar el desarrollo financiero de manera inclusiva y sostenible, como tampoco se ha analizado si las asimetrías de información han contribuido a ello. Iglesias (2008), señala que los 
procesos de inclusión financiera en el Perú se han visto favorecidos por el crecimiento económico mostrado por el Perú hasta el año 2018. Además, la mejora en el acceso, uso y calidad de los servicios financieros se plasman en la Política Nacional de Inclusión Financiera (DS 029-2014-EF, modificada por DS 255-2019-EF).

La evidencia señala por un lado que, se ha triplicado el número de clientes de las IMF, se ha triplicado el saldo de colocaciones (SBS, 2017) y se ha duplicado el número de Oficinas (BCP, 2017). Pese a este desenvolvimiento, el 50\% de las utilidades se concentraron en dos microfinancieras (una de ellas Caja Piura), y al menos 08 mostraron pérdidas entre 2016-2018 por deterioro en la calidad de la cartera (SBS, 2017). Según Valentín (2018), se hace necesario evaluar los desempeños de las IMF en las regiones del Perú como Piura, en donde se observan procesos de inclusión financiera frente al crecimiento de colocaciones y deterioros de la calidad de la cartera, particularmente por la marcada presencia de asimetrías de información.

De este análisis se infiere que, ante asimetrías informativas, las IMF podrían verse tentadas a endurecer sus políticas crediticias; ya sea evaluando con mayor rigurosidad la otorgación de créditos o disminuyendo los montos a colocar, lo cual afectaría a los deudores cumplidos. Las asimetrías, se expresarían en comportamientos minimizadores de riesgo por no contar con suficiente información del acreedor. Al mismo tiempo, el potencial sujeto crediticio podría creer no poder cumplir con los compromisos de pagos o no entender la real magnitud de la relación contractual que asume.

Por lo anterior, se hace necesario reflexionar sobre ¿Cómo abordar la asimetría de la información propia de procesos de inclusión financiera? ¿Cómo ha sido la discusión teórica de la asimetría de la información en las finanzas inclusivas? ¿Cómo se explica que pese al buen desempeño financiero de las IMF no se estaría asegurando la sostenibilidad social de las finanzas inclusivas desde los usuarios? ¿Qué rol juega en dicha dicotomía las asimetrías de información en el contexto regional de Piura?

El debate teórico de las microfinanzas inclusivas, señala a las microfinanzas como el conjunto de "prestaciones de servicios financieros formales para las personas pobres, de bajos ingresos, no asalariadas, y sistemáticamente excluidas del sistema financiero" (Banco Mundial, 2012). Al respecto, Infante y Chacaltana (2014), agregan que debido a que, el acceso a los sistemas bancarios tradicionales es limitado o inexistente en virtud 
de la condición socioeconómica de los involucrados, la exclusión se produce también, por los escasos o nulos niveles de educación.

Todo ello, hace que resulte poco o nada confiables y, por tanto, naturalmente excluidos del sistema financiero tradicional. Podemos observar que, las microfinanzas envuelven un aspecto innato de intermediación financiera e intermediación social; y por ello, se requiere de servicios financieros más que eficientes, fuertemente efectivos para compatibilizar el buen desempeño financiero de las IMF y el necesario desempeño social de las microfinanzas.

Infante y Chacaltana (2014) explican, desde el enfoque maximalista, que las microfinanzas enfatizan el impacto social directo de los servicios financieros en el mejoramiento de las condiciones sociales de los beneficiarios -salud, educación, participación social, vivienda, entre otras NBI-. Se trata de un instrumento de desarrollo que no puede ni debe ser evaluado desde su rentabilidad, sino desde su eficacia social. En esta perspectiva, las microfinanzas son inclusivas por naturaleza, se alinean a los Objetivos del Milenio para el Desarrollo (ODM); y su alcance se enfoca a atender necesidades económicas y financieras para abatir la pobreza, reforzar la autonomía y capacidad emprendedora; y asegurar impactos positivos sobre el nivel de vida de sus usuarios. Desde el enfoque minimalista, Iglesias (2018), sostiene la necesidad de crear condiciones de accesibilidad al crédito y ahorro para luego luchar contra la pobreza. Entre los enfoques maximalista y minimalista, el consenso es reforzar la conexión hogares-comunidad-mercado-estado y modificar los factores estructurales -sociales, económicos, políticos y legales- que impiden el acceso al capital financiero.

\section{Presencia de asimetrías de información en los mercados financieros inclusivos}

La literatura que aborda la presencia de asimetrías de información es amplia, pero Sánchez (2015), rescata la importancia de diferenciar entre incertidumbre e información asimétrica. La primera para referirse a información incompleta y la segunda para denotar información no disponible en el mercado. En las microfinanzas, esto es más que frecuente e inevitable por los riesgos morales y la selección adversa a la que puede conducir. El funcionamiento de las microfinanzas está muy lejos de la eficiencia paretiana, y las causas de ello, están en las externalidades, en los costos de transacción y en la imperfección de un mercado que pretende ser inclusivo pero que 
debe lidiar con un excluyente sistema financiero tradicional y un mercado financiero paralelo de 'usura' e informalidad.

Las asimetrías no pueden ser observadas ni cuantificadas mientras ocurren, pero son perceptibles cuando se producen efectos de riesgo e incertidumbre tanto para la IMF como para el usuario. Stiglitz (2002), sostiene que las transacciones tienen costos y transmiten información limitada o privilegiada que originan divergencias entre lo observado y lo esperado. En las finanzas, la asimetría de la información transgrede el riguroso supuesto de la eficiencia, la neutralidad al riesgo y el comportamiento optimizador de los agentes.

Las IMF normalmente obtienen conocimiento incompleto o limitado de la solvencia o calidad crediticia de los usuarios; y, por tanto, no siempre tienen suficiente capacidad para evaluar la calidad crediticia. La información asimétrica permite esconder aspectos del carácter y capacidad de pago; así como de la posición financiera del sujeto de crédito, aumentando el riesgo moral y afectando la probabilidad de pago ante selecciones adversas. La FAO (2002), el Banco Mundial (2012) y el BID (2018), aceptan que el micro financiamiento debe plantearse desde una perspectiva integral. Para ello, se requiere efectiva educación financiera, seguimiento y sostenibilidad.

Estamos ante una tendencia mundial que, desde el año 2010, más de 55 países asumieron el compromiso de implementar procesos de inclusión financiera y, más de 30 de ellos -incluido Perú- han puesto en marcha estrategias nacionales; como es el caso de México (Villarreal, 2017) y en América Latina (Villacorta \& Reyes, 2012) siempre en la línea del Banco Mundial (2008). A nivel de América Latina y el Caribe (CEPAL, 2016), se muestra bajo y desigual acceso al sistema financiero, y se verifican marcadas brechas relacionadas con ingresos, género y regiones. Entonces, la reflexión nos conduce a preguntarnos: ¿Es posible mostrar buen desempeño financiero y al mismo tiempo promover procesos inclusivos sociales? ¿Cómo afectan las asimetrías de información a este proceso? ¿Cómo ha sido dicha conexión en base a la evidencia de Piura? La tabla 1, muestra el planteamiento que sustenta este trabajo.

El análisis de la información asimétrica, según Sánchez-Daza (2015) surge de la Hipótesis de los Mercados Eficientes y, de sus fallas que hace que se manifieste como fracaso del mercado. Esta falla facilita el surgimiento de transmisores inadecuados de información o noticias acerca de la calidad y perfil crediticio de los usuarios. De 
hecho, la información asimétrica supone que una de las partes contractuales tiene menos información que otras, derivando con frecuencia en selección adversa, riesgo moral y comportamientos de rebaño. Todo este mecanismo es sintetizado en la figura 1 .

Figura 1: Información asimétrica en procesos financieros inclusivos

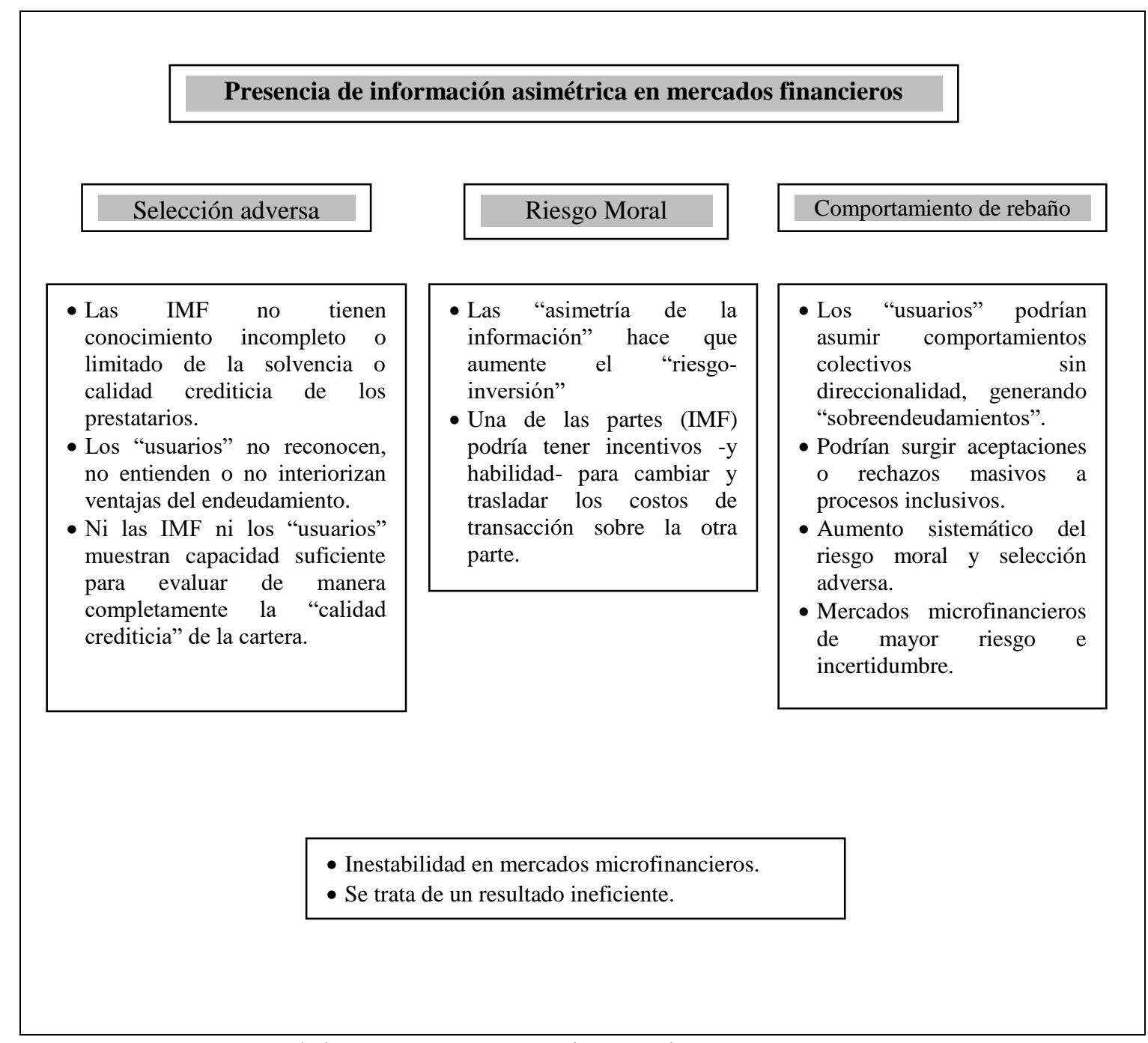

Elaboración propia en base a documentos varios.

De la figura 1, se infiere que el análisis de información asimétrica en procesos financieros inclusivos se soporta en Stiglitz (2002). Las IMF buscan maximizar colocaciones seleccionando usuarios que cumplan condiciones de carácter, capacidad de pago y mínima posición financiera aceptable. Probablemente, es más factible en usuarios involucrados en actividades laborales formales, con algún nivel de educación financiera o no-, y con alguna experiencia o historial crediticio; pero además dispuestos a incorporarse a estos procesos. Estos elementos pueden y deben ser modelados. 


\section{ESTRATEGIAS METODOLÓGICAS, MATERIALES Y MÉTODOS}

Las estrategias metodológicas seguidas se orientan a explicar el buen desempeño de la IMF y la posibilidad de participar o no en procesos financieros inclusivos; así como las decisiones e interacciones que ocurren entre ellas, las cuales, bien podrían no reflejar de manera clara las asimetrías de información que las involucra (Tabla 1).

Tabla 1: Desempeño Financiero y Social de las IMF con asimetría de información

\begin{tabular}{|c|c|c|c|}
\hline Desempeño & Planteamiento & Planteamiento & Hipótesis a probar \\
\hline Financiero & $\begin{array}{l}\text { ¿Cómo ha sido el } \\
\text { desempeño } \\
\text { financiero de las } \\
\text { IMF que operan en } \\
\text { escenarios de } \\
\text { asimetrías } \\
\text { informativas? }\end{array}$ & $\begin{array}{l}\text { ¿Cuáles son } \\
\text { factores } \\
\text { determinan el buen } \\
\text { desempeño de las IMF } \\
\text { en escenarios } \\
\text { asimétricos? }\end{array}$ & $\begin{array}{l}\text { En escenarios de asimetría } \\
\text { informativa, las IMF a pesar } \\
\text { del buen desempeño } \\
\text { financiero muestran deterioro } \\
\text { en la calidad de su cartera. }\end{array}$ \\
\hline Social & $\begin{array}{l}\text { ¿Cómo ha sido el } \\
\text { desempeño social de } \\
\text { las microfinanzas } \\
\text { inclusivas } \\
\text { presencia } \\
\text { asimetrías } \\
\text { información? }\end{array}$ & $\begin{array}{l}\text { ¿Qué variables } \\
\text { socioeconómicas } \\
\text { inducen a escenarios } \\
\text { de microfinanzas } \\
\text { inclusivas } \\
\text { escenarios } \\
\text { información } \\
\text { asimétrica? }\end{array}$ & $\begin{array}{l}\text { En presencia de asimetría de } \\
\text { información las variables } \\
\text { socioeconómicas, como el } \\
\text { nivel educativo, la situación } \\
\text { laboral, la experiencia } \\
\text { crediticia y la edad del } \\
\text { potencial usuario determinan } \\
\text { la posibilidad de incorporarse } \\
\text { en procesos financieros } \\
\text { inclusivos. }\end{array}$ \\
\hline
\end{tabular}

Elaboración propia

La presencia de asimetrías de información sesga las razones por las cuales un potencial usuario pueda reconocer la posibilidad de incorporarse a procesos financieros inclusivos. Incluso las IMF, en estos mismos escenarios, bien podrían mostrar buen desempeño financiero, pero con deterioro en la calidad de cartera. Las asimetrías afectan la probabilidad de incorporarse en dicho proceso inclusivo y mantenerse en él. La inclusión financiera es mucho más que el uso o acceso a servicios financieros; en realidad, es la respuesta a un fallo de mercado y como tal, un desafío social. 


\section{a. Modelación}

Para el análisis del desempeño financiero de las IMF, se considera que el buen desempeño financiero no garantiza adecuado desempeño social debido a la presencia de asimetrías informativas. En la línea de Gutiérrez y Goiria (2015) se propone el análisis factorial para evaluar el desempeño financiero, mientras que para el desempeño social se propone un modelo de respuesta binaria (logit). El uso del análisis factorial se plantea para reconocer los componentes del desempeño financiero en IMF que operan en mercados con fuerte componente de asimetría; se parte de la metodología seguida por Sánchez-Daza (2015), quien aplicando análisis factorial muestra evidencia de la relación existente entre variables representativas de los desempeños social y financiero. A partir de ello, se hace necesario evaluar el conflicto entre lo social y lo financiero. De la misma manera, y en la línea de López-Iturraga (1996), se aborda la relación entre estos desempeños. A partir de ello, se plantea que:

$X_{p}=a_{p 1} F_{1}+a_{p 2} F_{2}+\ldots+a_{p k} F_{k}+U_{p}$

$X_{p}=$ Es el valor de la " $p$-ésima" variable involucrada

$\mathrm{a}_{\mathrm{pk}}=$ Son las "puntuaciones factoriales" o "cargas factoriales"

$\mathrm{F}_{\mathrm{k}}=$ Son los "coeficientes factoriales (factores comunes)

$\mathrm{U}_{\mathrm{p}}=$ Factor único o específico correspondiente a la "p-ésima" variable.

Se propone el análisis factorial como técnica estadística multivariante, para sintetizar información a partir de varios elementos observados, un número reducido de nuevas variables denominadas factores o componentes. Se aplica a una matriz de datos con $\boldsymbol{n}$ individuos y $\boldsymbol{p}$ variables, mediante $\boldsymbol{k}$ factores, siendo $\boldsymbol{k}<\boldsymbol{p}$. Este proceso se operativizará en SPSS; y es útil cuando no se tiene un punto de partida que identifique componentes para explicar el desempeño y sostenibilidad financiera de una IMF.

Para el análisis del desempeño social de las IMF, se propone el uso de un modelo de respuesta binaria (logit); en este caso, para reconocer el desempeño social en escenarios de asimetrías informativas. Se ha determinado la existencia de variables que explican la inclusión financiera, en ellas destacan: edad, sexo, estado civil, nivel educativo, zona de residencia e ingreso; con la finalidad de aproximarnos a la probabilidad de ocurrencia de si se es parte de la inclusión financiera vs no se es parte de la inclusión financiera. 
La regresión binaria reconoce como variable dependiente $\boldsymbol{Z}_{i}=\mathbf{1}$ para el primer caso y $Z_{i}=1$ para la segunda situación. La ventaja de aplicar modelación logit es que permite replantear -si fuera el caso- nuevas hipótesis; en este caso luego de reconocer o no si el buen desempeño de las IMFs que operan en Piura está referido a logros de sostenibilidad. Para tal fin, se plantea que:

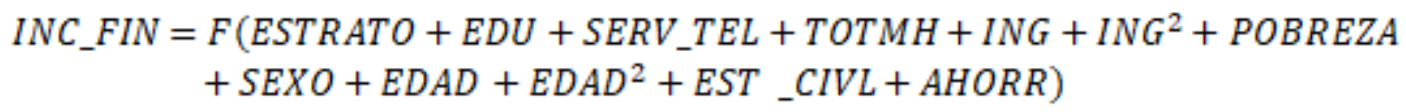

Donde INC_FIN representa la inclusión financiera en escenarios de asimetría de información, la cual puede ser medida por el acceso a algún servicio financiero. Las variables independientes reflejan factores que determinarían los escenarios asimétricos en el cual los agentes de Piura se incorporan a procesos financieros inclusivos.

\section{b. Tratamiento de datos}

Las tablas 2 y 3, presentan la ruta metodológica seguida en el presente estudio. La identificación del estudio se basó en integrar los aspectos del desempeño financiero y desempeño social de las microfinanzas, pues ambos deben configurarse en escenarios de asimetría informativa. En primer lugar, para mostrar que las IMF a pesar del buen desempeño financiero muestran deterioro en la calidad de su cartera; y que en presencia de asimetría de información las variables socioeconómicas, como el nivel educativo, la situación laboral, la experiencia crediticia y la edad del potencial usuario determinan la posibilidad de incorporarse en procesos financieros inclusivos. Los signos que se espera de en cada variable aparecen ver en el Anexo 3, en donde también se presentan las particularidades metodológicas de este estudio.

La data pertinente para esta investigación, fue estructurada a partir de las bases de datos de ENAHO (2019), la cual permitió las estimaciones aplicando análisis factorial y modelación logit. La aplicación de estas técnicas, se plantean para mostrar ¿Cuáles serían los factores que mejor reflejan el desempeño financiero de las IMF que operan enfrentando el fallo de la asimetría de información? La revisión teórica realizada respalda la idea de que dichas asimetrías se manifiestan en decisiones de selección adversa y aumento en el riesgo moral, lo cual no solo facilita el racionamiento de fondos, sino que, además, aumenta el riesgo y la tenencia de carteras de alto riesgo. 


\section{RESULTADOS Y DISCUSIÓN}

Los resultados de la investigación señalan que, en escenarios no asimétricos, las IMF deben orientar recursos locales para financiar iniciativas locales; pero ello, no es suficiente para garantizar su solvencia y sostenibilidad, por lo que en base al análisis factorial realizado, se encontró que para el caso de las IMF que ofrecen servicios financieros inclusivos, si bien es cierto -a pesar de los escenarios asimétricos de información- logran buen desempeño financiero, muestran débiles calidades de carteras, debido a que los usuarios de los servicios crediticios no siempre asumen comportamientos acordes con un buen desempeño social.

Se encontró que, entre los factores más relevantes que explican la sostenibilidad de las IMF está la solvencia, la calidad de la cartera o morosidad normal, la calidad de la cartera o "cartera de alto riesgo", la gestión y la eficiencia de la IMF. En base a ello y a partir de la base de datos disponible en la SBS (2019), se definieron operacionalmente dichas variables, como se muestra en la tabla 2 .

Tabla 2: Indicadores de Desempeño Financiero de las IMF: Perú

\begin{tabular}{|l|l|}
\hline \multicolumn{1}{|c|}{ VARIABLES } & \multicolumn{1}{c|}{ INDICADOR } \\
\hline SOLVENCIA & Pasivo Total / Capital Social y Reservas ( $\mathrm{N}^{\circ}$ de veces ) \\
\hline CALIDAD CARTERA1 & Créditos Atrasados (criterio SBS)** / Créditos Directos \\
\hline CALIDAD CARTERA2 & Cartera de Alto Riesgo / Créditos Directos (\%) \\
\hline GESTIÓN & Créditos Directos / Número de Oficinas (Miles S/) \\
\hline EFICIENCIA & Utilidad Neta Anualizada sobre Patrimonio Promedio (\%) \\
\hline
\end{tabular}

Elaboración propia.

Para el análisis factorial, se seleccionó 05 indicadores financieros de 18 IMF, todos estandarizados en ratios para facilitar su comparación. La solvencia fue medida por la relación entre pasivos totales y capital social -incluyendo reservas- y expresado en número de veces. Debido a la presencia de asimetrías y por la alta probabilidad de riesgo moral, se explora si los indicadores de cartera -morosidad- relacionan colocaciones atrasadas frente a créditos directos. Igualmente, incluye la cartera de alto riesgo, variables afectadas por los entornos adversos de información confiable.

La variable gestión, se seleccionó como componente probable para explicar el desempeño financiero de las IMF, permite comparar los créditos directos con el número de oficinas que operan en Piura. Ésta no es la única forma de referir la capacidad de 
gestión, pero constituye una medida estándar que permite comparar las colocaciones directas por oficina. La eficiencia fue medida desde la forma óptima de asignar las inversiones (activos) o retorno que ofrecen las inversiones financiadas con recursos patrimoniales. Se trata de un adecuado indicador de eficiencia, cuyo tratamiento se presenta en la tabla 3. El tratamiento de los datos que operacionalizan las variables comprendidas en el modelo logit, se contextualiza en base a las estadísticas descriptivas que se muestran en la tabla 6; en donde se observa alta variabilidad de los indicadores de ingresos familiares y la casi inexistente capacidad de ahorro.

Tabla 3: Indicadores de Desempeño Financiero de las IMF: Perú

\begin{tabular}{|l|l|c|c|c|c|c|}
\hline $\mathbf{N}^{\circ}$ & \multicolumn{1}{|c|}{ IMF } & Solvencia & Calid cart 1 & Calid cart 2 & Gestión & Eficiencia \\
\hline $\mathbf{1}$ & Caja Arequipa & 8.44 & $4 . .55$ & 6.67 & 29,743 & 19.54 \\
\hline $\mathbf{2}$ & CMAC Cusco & 6.87 & 4.44 & 5.72 & 28,274 & 16.06 \\
\hline $\mathbf{3}$ & CMAC Santa & 5.99 & 22.92 & 26.77 & 10,245 & -8.99 \\
\hline $\mathbf{4}$ & CMAC Huancayo & 7.65 & 3.52 & 4.57 & 24,328 & 19.52 \\
\hline $\mathbf{5}$ & CMAC Ica & 6.24 & 5.3 & 6.48 & 22,833 & 12.74 \\
\hline $\mathbf{6}$ & CMAC Maynas & 5.19 & 6.57 & 8.9 & 19,245 & 8.68 \\
\hline $\mathbf{7}$ & CMAC Paita & 4.36 & 13.6 & 17.34 & 9,334 & 0.4 \\
\hline $\mathbf{8}$ & CMAC Piura & 9.84 & 6.91 & 9.17 & 20,379 & 13.47 \\
\hline $\mathbf{9}$ & CMAC Sullana & 10.41 & 15.35 & 21.43 & 29,361 & -14.95 \\
\hline $\mathbf{1 0}$ & CMAC Tacna & 6.84 & 6.76 & 9.95 & 27,809 & 7.59 \\
\hline $\mathbf{1 1}$ & CMAC Trujillo & 4.48 & 5.95 & 7.41 & 19,064 & 7.12 \\
\hline $\mathbf{1 2}$ & CMPCP Lima & 4.47 & 16.58 & 22.17 & 10,330 & -16.17 \\
\hline $\mathbf{1 3}$ & CRAC Raíz & 5.53 & 7.39 & 10.16 & 17,805 & 3.16 \\
\hline $\mathbf{1 4}$ & CRAC Sipán & 1.52 & 8.59 & 10.38 & 8,816 & -10.6 \\
\hline $\mathbf{1 5}$ & CRAC Los Andes & 10.81 & 6.77 & 9.65 & 7,507 & 15.96 \\
\hline $\mathbf{1 6}$ & CRAC Primera & 3.18 & 5.87 & 8.95 & 9,225 & -19.46 \\
\hline $\mathbf{1 7}$ & CRAC Incasur & 3.02 & 7.25 & 7.91 & 12,725 & 1.69 \\
\hline $\mathbf{1 8}$ & CRAC del Centro & 4.26 & 2.97 & 4.66 & 30,031 & -18.06 \\
\hline
\end{tabular}

Elaboración propia - Fuente: $\underline{\text { https://www.sbs.gob.pe }}$

Al aplicar el análisis factorial a los componentes del desempeño financiero, se logra un proceso de reducción de datos utilizando correlaciones entre variables observadas y factores o componentes, considerando su dispersión, correlación y normalidad. A partir 
de este análisis exploratorio se reconoce la estructura interna de variables que explican el desempeño financiero de un conjunto de IMF. Las pruebas KMO y Barlett (Tabla 4), muestran aceptable Coeficiente de confiabilidad estadística.

Tabla 4: Prueba de KMO y Barlett

\begin{tabular}{|l|l|c|}
\hline \multicolumn{2}{|c|}{ Medida Kaiser-Meyer-Olkin de adecuación de muestreo } & 0.540 \\
\hline \multirow{3}{*}{ Prueba de esfericidad de Barlett } & Aproximación Chi ${ }^{2}$ & 32.329 \\
\cline { 2 - 3 } & Grados de Libertad (gl) & 10 \\
\cline { 2 - 3 } & Significancia (Sig.) & 0.00 \\
\hline
\end{tabular}

Elaboración propia - Estimaciones en SPSS 25.0

El análisis de varianza total explicada (tabla 5) muestra los autovalores y sus dispersiones, las mismas que se calculan bajo el método de extracción de máxima verosimilitud.

Tabla 5: Varianza total explicada

\begin{tabular}{|c|c|c|c|c|c|c|c|c|c|}
\hline & \multirow{2}{*}{ Factor } & \multirow{2}{*}{$\begin{array}{c}\text { \% de } \\
\text { Total }\end{array}$} & \multirow{2}{*}{$\begin{array}{c}\text { \% } \\
\text { Varianza }\end{array}$} & \multicolumn{2}{|c|}{$\begin{array}{c}\text { Sumas de cargas al cuadrado } \\
\text { acumulado la extracción }\end{array}$} & \multicolumn{3}{|c|}{$\begin{array}{c}\text { Sumas de cargas al cuadrado } \\
\text { de la rotación }\end{array}$} \\
\cline { 5 - 10 } & & & Total & $\begin{array}{c}\text { \% de } \\
\text { Varianza }\end{array}$ & $\begin{array}{c}\text { \% } \\
\text { acumulado }\end{array}$ & Total & $\begin{array}{c}\text { \% de } \\
\text { Varianza }\end{array}$ & $\begin{array}{c}\text { \% } \\
\text { acumulado }\end{array}$ \\
\hline 1 & 2.479 & 49.586 & 49.586 & 1.362 & 27.248 & 27.248 & 2.011 & 40.217 & 40.217 \\
\hline 2 & 1.357 & 27.145 & 76.732 & 2.118 & 42.365 & 69.613 & 1.470 & 29.396 & 69.613 \\
\hline 3 & 0.737 & 14.733 & 91.465 & & & & & & \\
\hline 4 & 0.260 & 5.192 & 96.657 & & & & & & \\
\hline 5 & 0.167 & 3.343 & 100.00 & & & & & & \\
\hline
\end{tabular}

Método de extracción: máxima verosimilitud

De acuerdo a este resultado, la cartera de alto riesgo y el riesgo crediticio reflejan las asimetrías de información que las IMF enfrentan en el mercado micro financiero piurano. Este mismo resultado se observa en el gráfico de sedimentación (figura 3).

Figura 2: Gráfico de sedimentación

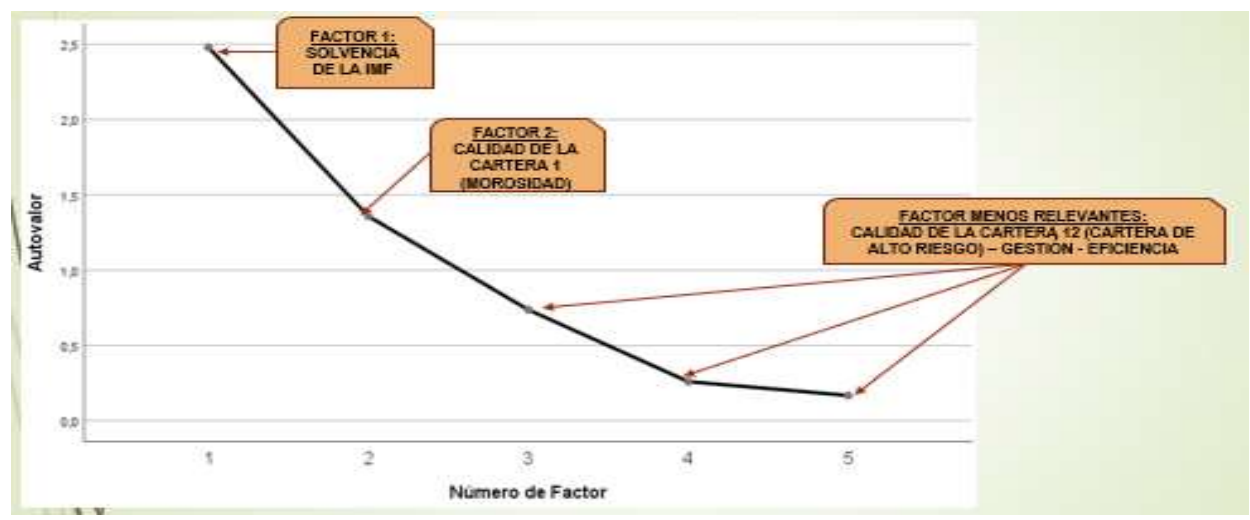

Elaboración propia-SPSS 25.0

Ciencia Latina Revista Científica Multidisciplinar, Ciudad de México, México. ISSN 2707-2207 / ISSN 2707-2215 (en línea), mayo-junio, 2021, Volumen 5, Número 3. https://doi.org/10.37811/cl_rcm.v5i3.563 p. 3795 
Según la figura 2, se logra representar la magnitud e importancia de los autovalores en el problema de inclusión a explicar. Esto, ayuda a identificar el número óptimo de factores que se deben considerar; y aunque puede ser permite $\mathrm{r}$ reconocer que los "autovalores" que mejor explican la mayor parte de la varianza se ubican en la parte izquierda del gráfico; es decir, la solvencia y la calidad de la cartera entendida como morosidad. Los resultados de estimación logit se presentan en la tabla 6.

\section{Tabla 6: Estimación del modelo logit}

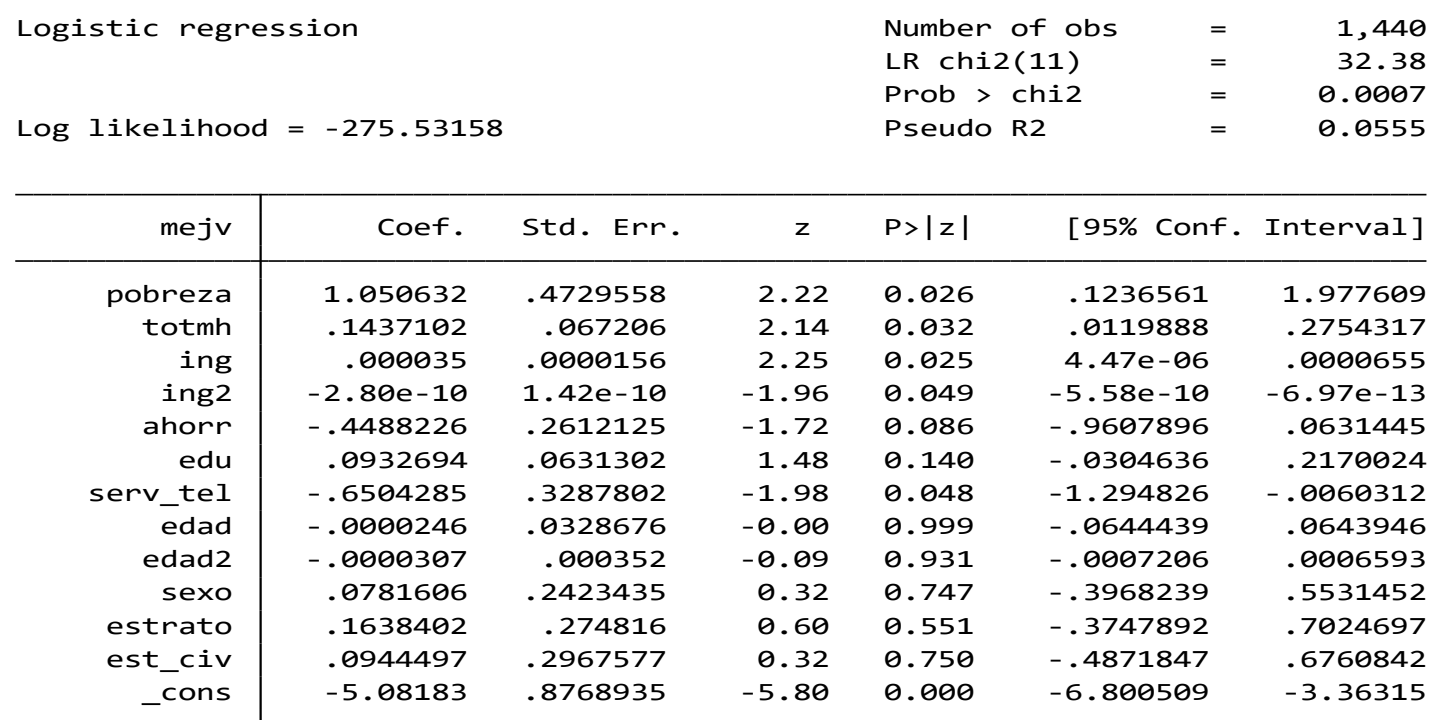

\section{Elaboración en base a ENAHO 2019 - INEI}

Por su parte, en base a los datos de la encuesta ENAHO (2019), luego de varias estimaciones logit, se optó por considerar 12 variables explicativas de las 22 variables propuestas. Las estimaciones con todas las variables explicativas no eran congruentes con los signos esperados de los parámetros y, además, los estadísticos no eran significativos tanto de manera individual como conjuntamente. En base a estas estimaciones, se presenta un modelo logit ya que presenta mejores estadísticos. La estimación se realizó con el software STATA 15,0 para 1440 hogares.

La evaluación económica, permite observar que los resultados obtenidos en el modelo, que se cumplen las relaciones teóricas esperadas. Se verifica que, a medida que aumenta la edad, en los primeros años el impacto sobre la inclusión financiera es positiva, por ende, creciente, sin embargo, a medida que aumentan los años el impacto es negativo, decreciente. Es decir, cuanto más adulto es un individuo menores posibilidades de acceder al sistema financiero tienen, reduce la demanda de servicios financieros. 
A la adultez, los individuos optan por atender sus necesidades básicas, como alimentación y salud. Por consiguiente, mayores niveles de educación implica mayor acceso al sistema financiero; por lo que se confirma la importancia de la educación para incrementar el acceso a los servicios financieros. Sin embargo, la educación sería un mejor detonante de los procesos financieros inclusivos si es que se orientan al desarrollo de capacidades en el contexto de una efectiva educación financiera, pues ello, reduciría las asimetrías de información desde el punto de vista del usuario.

Con respecto a los ingresos, se observa que existe una relación positiva con la inclusión financiera, debido a que un mayor nivel de ingresos implica mayores probabilidades de que los individuos accedan a los servicios financieros, por ende, se incrementa la inclusión financiera en la región. El factor sexo, muestra que los hombres son quienes tienen mayor probabilidad de acceder al sistema financiero, lo cual puede deberse a la posición de jefe de familia que asume desde la base muestral. También se encuentra que los niveles de pobreza implican mayor necesidad de incorporarse al sistema financiero, aunque ello aumenta la probabilidad de fracaso en la capacidad de pago.

La evaluación estadística y econométrica del modelo estimado no presenta problemas de heterocedasticidad, multicolinealidad ni autocorrelación, lo cual ha sido comprobado con los test de Siegel- Tukey. Estos test muestran la presencia de homocedasticidad; mientras que la evaluación de autocorrelación -mediante autocorrelograma- evidenció que todos los datos y valores del modelo se encuentran dentro de la banda de ajuste; mientras que, la multicolinealidad, se analizó mediante el gráfico de la elipse donde se puede observar que, no hay colinealidad entre las variables.

De estos resultados se desprende que los procesos microfinancieros inclusivos de Piura están supeditados a escenarios en donde las asimetrías de la información están fuertemente presentes. Las imperfecciones de los mercados financieros locales se centran en serias dicotomías que nos lleva a reflexionar acerca de si las finanzas inclusivas son en realidad un proceso maximilista en donde la búsqueda de la mejor cartera de colocaciones y de servicios financieros eficientes; o en realidad se trata de un proceso más integral en donde el desempeño microfinanciero, por sí mismo, no tiene relevancia, sino no es evaluado en el contexto de un proceso social, inclusivo.

$\mathrm{Si}$ fuera lo segundo, debe aceptarse entonces que como proceso social, las microfinanzas nacen, y son respuestas a las fallas de mercados financieros; las cual se 
manifiestan en lo que se conoce como selección adversa, riesgo moral y efecto rebaño; que lo único que lograr es acentuar las brechas sociales, se alejan del propósito de constituir capital financiero y no estarían contribuyendo a la sostenibilidad de las finanzas locales.

Debido a las asimetrías de información, y pese al buen desempeño de las IMF -desde el punto de vista de la gestión, solvencia y rentabilidad- se observa deterioro de la calidad de la cartera. No obstante, desde el desempeño social de las finanzas inclusivas lideradas por las IMF en Piura, no se estaría reflejando procesos financieros inclusivos fuertes y sostenibles debido a la presencia de asimetrías que no han sido suficientemente evaluadas desde los componentes que determinan el buen desempeño financiero de las IMF, al mismo tiempo que no se han reconocido los factores que determinan la posibilidad y pertinencia de incorporarse al referido proceso financiero inclusivo.

Los resultados del estudio comprueban que, en efecto, la hipótesis de Stiglitz (2002) sobre la asimetría de información afectan de manera contraria la probabilidad de incorporarse a procesos financieros inclusivos, motivando restricciones en las colocaciones y se contraiga el ahorro.

Para el caso de Piura, se encuentra que desde el análisis factorial se observa un buen desempeño de las IMF como organizaciones que buscan la sostenibilidad basada en estrategias de solvencia, adecuado manejo de la calidad de las carteras y eficiencia. Las IMF necesitan de carteras sanas y requieren de ampliar el acceso a capital financiero y ser eficientes en el proceso.; pero ello, debido a la presencia de asimetrías de información no siempre sucede así, por lo que la calidad de la cartera y la morosidad reflejan esos altos riesgos.

Ante escenarios de asimetría de información, no se puede afirmar que la búsqueda de la gestión, entendida como ampliación de las colocaciones por agencia ni la rentabilidad patrimonial, son condiciones suficientes para lograr la inclusión financiera, aunque bien pudieran ser condiciones necesarias; es decir importa, pero no estarían determinando la naturaleza inclusiva de las microfinanzas.

Por otro lado, la estimación logit muestra que, para efectos de estudio se postuló una hipótesis general central de investigación. Un determinante que influye de manera positiva en un primer momento, o estadio de tiempo, es la variable ingreso, debido a que incrementa la probabilidad de inclusión financiera, es decir, un mayor poder 
adquisitivo conlleva a que el individuo acceda a los servicios financieros como créditos, depósitos u otros.

\section{CONCLUSIÓN Y CONSIDERACIONES FINALES}

En Piura, se verifican procesos financieros inclusivos con marcados escenarios de asimetría de información, en donde las microfinanzas han sido una clara respuesta a estos fallos de mercado. Los procesos de inclusión financiera, son necesariamente procesos sociales y no puramente procesos financieros, pues están orientados a generar mejores condiciones de vida para los usuarios de servicios financieros inclusivos. Estos procesos se han mostrado débiles e insuficientes, aunque claramente conectados a los niveles de educación y la formalidad en las actividades laborales que realizan las familias piuranas.

La estimación de regresión logarítmica binaria muestra que es posible predecir procesos de inclusión financiera en escenarios de asimetría de información, hasta en un 69.6\% de predictibilidad; lo cual da señales que existen otras variables predictoras que van más allá de la edad (18-50 años) y más allá de la experiencia laboral de las familias de Piura. Se concluye que, el factor más importante del proceso de inclusión es la solvencia de las IMF, junto con el control de la morosidad -normal y de alto riesgo-. Al parecer, la gestión y la rentabilidad, son indicadores de sostenibilidad interna de las IMF, que importan, pero no determinan los deseados procesos de inclusión financiera.

Las microfinanzas inclusivas están sujetas a asimetrías de información por la relación de fuerzas desiguales, y sujetas a evaluación de sus impactos multidimensionales. Por ello, el papel del estado en materia de microfinanzas inclusivas y en entornos asimétricos, debe configurarse como el de promotor y colaborador del funcionamiento armónico de los mercados. Su labor debe estar orientada a proveer adecuados sistemas regulatorios, que busquen reducción de los costos de transacción del mercado.

Por otro lado, nuevas pistas de investigación surgen de este estudio. En escenarios asimétricos, las IMF reaccionan con estrategias de racionamiento para minimizar riesgos. La probabilidad de que una IMF logre procesos financieros inclusivos y sostenibles podría ser un objetivo contradictorio con su objetivo de desempeño financiero. Esta hipótesis amerita investigación profunda, pues todo parece indicar que la inclusión financiera está marcada por fuertes asimetrías, y por su naturaleza social merece respuestas de política por parte del estado. 


\section{LISTA DE REFERENCIAS}

Banco Mundial (2012) Guía para la regulación y la supervisión de las Microfinanzas: Directrices de consenso. Washington, DC.

BCRP (2017, 2018, 2019). Microfinanzas y crecimiento económico. En: https://www.eventosfpcmac.com/wp-content/uploads/2019/09/S0-InaguracionMichel-Canta-Terreros-MEF.pdf

BID (2018). Microfinance. En: https://www.eventosfpcmac.com/wpcontent/uploads/2019/09/S0-Inaguracion-Michel-Canta-Terreros-MEF.pdf

CEPAL, P.L (2016). Inclusión financiera de las PYMES en el Ecuador y México. Naciones Unidos.

Política Nacional de Inclusión Financiera. DS 029-2014-EF modificado con DS 2552019-EF.

ENAHO (2019). Encuesta Nacional a Hogares. Perú. En: https://www.inec.cr/multimedia/enaho-2019-presentacion-de-la-encuestanacional-de-hogares-2019

FAO (2002) Servicios bancarios para los pobres: El microcrédito no basta, los sectores pobres necesitan otros servicios financieros Disponible en: http://www.fao.org/worldfoodsummit/spanish/newsroom/focus/focus5.htm

Gutierres-Goiria J. (2015). Las microfinanzas y su evaluación social. Universidad del País Vasco (UPC-EHU). En: https://www.academia.edu/28264166/LAS_MICROFINANZAS_Y_SU_EVAL UACI\%C3\%93N_SOCIAL_FUENTE_DE_APRENDIZAJE_PARA_LA_RSE

Iglesias, María F. (2008) “¿Cómo potenciar el impacto social de las microfinanzas? El enfoque integral vs. el enfoque minimalista. El rol del Estado"-Centro de Estudios de la Estructura Económica-Universidad de Buenos Aires.

Infante y Chacaltana (2014). Editores. Hacia un desarrollo inclusivo, el caso de Perú. Publicación de las Naciones Unidas- LC/L.3779 - CEPAL - OIT

López-Iturriaga (1996). La asimetría informativa en los mercados financieros: ¿el hallazgo de un nexo de unión. Universidad de Valladolid.

Sánchez (2015). Información asimétrica en el mercado de capitales: Colombia. Pontificia Universidad Javeriana - Bógota, Colombia. Disponible en: https://www.researchgate.net/publication/2857634 
Sánchez-Daza (2015). Información asimétrica y mercados financieros emergentes: el análisis de Mishkin. Revista Análisis Económico - Departamento de Economía de la UAM-Azcapotzalco (sánchezdaza@yahoo.com). México.

SBS (2019). Superintendencia de Banca y Seguros. En: https://www.sbs.gob.pe/regulacion/plan-de-cuentas/sistemafinanciero/microfinancieras-no-bancarias

Striglitz J (2002). La información y cambio en el paradigma de la ciencia económica. Universidad de Columbia. Revista Asturiana de Economía. RAE N²5- 2002.

Valentin M (2017). Desarrollo local y microfinanzas como estrategias de atención a las necesidades sociales: un acercamiento teórico conceptual. Disponible en: http://creativecommons.org/licenses/by-nc-nd/4.0/

Villacorta, O. y Reyes JD. (2012). Servicios financieros para las mayorías. La inclusión financiera en México. Revista Microfinanzas y Banca Social.

Villarreal, F.G. (2017). Inclusión financiera de pequeños productores rurales. En: Libros de la CEPAL, N 147 (LC/PUB.2017/15-P). 


\section{ANEXOS}

Anexo A1: Medición y Signos de las Variables Independientes

\begin{tabular}{|c|c|c|}
\hline $\begin{array}{l}\text { Variable } \\
\text { Dependiente }\end{array}$ & Medición & Signo \\
\hline \multicolumn{3}{|c|}{ Variable dependiente } \\
\hline INC_FIN & 1: $\mathrm{Si} \quad 0: \mathrm{No}$ & \\
\hline \multicolumn{3}{|c|}{ Variables Independientes } \\
\hline ESTRATO & 1: Urbano y 0: Rural & Positivo \\
\hline EDU & $\begin{array}{l}\text { 1: Sin nivel, 2: Educación inicial, 3: Primaria } \\
\text { incompleta, 4: Primaria completa, 5: Secundaria } \\
\text { incompleta, 6: Secundaria completa, 7: Superior } \\
\text { no universitaria incompleta, 8: Superior No } \\
\text { Universitaria completa, 9: Superior Universitaria } \\
\text { incompleta, 10: Superior Universitaria completa, } \\
\text { 11: Maestría/doctorado. }\end{array}$ & Positivo \\
\hline SERV_TEL & 1: Sí y 0: No & Positivo \\
\hline TOTMH & Número de miembros del hogar & Positivo \\
\hline ING & Cantidad de dinero & Positivo \\
\hline$I N G^{2}$ & Cantidad de dinero al cuadrado & Negativo \\
\hline POBREZA & 1: No Pobre y 0: Pobre & Positivo \\
\hline SEXO & 1: Hombre y 0: Mujer & Positivo \\
\hline EDAD & Edad en años & Positivo \\
\hline$E D A D^{2}$ & Edad en años al cuadrado & Negativo \\
\hline EST_CIVL & 1: Casado(a) y 0: Soltero(a) & Positivo \\
\hline AHORR & $\begin{array}{l}\text { 1: Cuenta con una cuenta de ahorro en alguna } \\
\text { institución financiera y } \\
\text { 0: No Cuenta con una cuenta de ahorro en alguna } \\
\text { institución financiera }\end{array}$ & Positivo \\
\hline
\end{tabular}


Anexo A2: Diseño metodológico para análisis empírico de microfinanzas inclusivas en Piura

\begin{tabular}{|c|c|c|}
\hline Aspecto & Descripción & Alcance \\
\hline $\begin{array}{l}\text { Tipo de } \\
\text { investigación }\end{array}$ & $\begin{array}{l}\text { Empírica: Se busca explicar la inclusión } \\
\text { financiera como respuesta al fallo de mercado: } \\
\text { asimetría de información. Se plantea un modelo } \\
\text { econométrico de respuesta binaria, por lo que se } \\
\text { trata de un estudio de corte transversal en base a } \\
\text { ENAHO (2019); y de un análisis de desempeño } \\
\text { financiero en base a un análisis factorial aplicado } \\
\text { a una muestra de 15 IMF que operan en Piura, en } \\
\text { base a la SBS (2019). }\end{array}$ & $\begin{array}{l}\text { Para el análisis del } \\
\text { desempeño social como } \\
\text { financiero de las micro } \\
\text { finanzas inclusivas se } \\
\text { considera la necesidad } \\
\text { de abordarlos } \\
\text { empíricamente y a la luz } \\
\text { de la teoría y evidencia. }\end{array}$ \\
\hline $\begin{array}{l}\text { Diseño de } \\
\text { Investigación }\end{array}$ & $\begin{array}{l}\text { Diseño mixto. Incorpora elementos descriptivos, } \\
\text { probabilísticos, cualitativo-cuantitativos y } \\
\text { causales. Involucra variables de hasta } 8 \\
\text { características y variables independientes } \\
\text { cuantitativas. La variable dependiente cualitativa } \\
\text { es binaria y refleja presencia de asimetrías que } \\
\text { afectan la probabilidad de incorporarse o no en } \\
\text { procesos de inclusión financiera. Se propone } \\
\text { aproximación logit y se recurre al software } \\
\text { STATA 15,0. Para el caso del análisis factorial } \\
\text { para aproximarnos al desempeño financiero, se } \\
\text { recurre al análisis factorial desde el SPSS } 20,0 \text {. } \\
\text { Ambas en versión libres y de prueba. }\end{array}$ & $\begin{array}{l}\text { Desempeño social de } \\
\text { IMF se aproxima desde } \\
\text { los determinantes socio- } \\
\text { económicos del proceso } \\
\text { de inclusión financiera } \\
\text { en entornos asimétricos } \\
\text { de información. El } \\
\text { desempeño financiero } \\
\text { busca reconocer } \\
\text { determinantes de solidez } \\
\text { y sostenibilidad de IMF. }\end{array}$ \\
\hline Muestra & $\begin{array}{l}\text { La base de datos es ENAHO (2019). El análisis } \\
\text { de los determinantes de la "Inclusión } \\
\text { Financiera en escenarios asimétricos", se } \\
\text { estimó a través de un modelo logit, teniendo } \\
\text { como punto de estudio la región de Piura para el } \\
\text { periodo } 2019 \text {. De la revisión teórica se adaptaron } \\
\text { las variables y se operacionalizaron, a partir de } \\
\text { Módulos } 01 \text { (Características de la Vivienda y del } \\
\text { Hogar), } 02 \text { (Características de los Miembros del } \\
\text { Hogar), } 03 \text { (Educación), 05 (Empleo e Ingresos), } \\
\text { y } 34 \text { (Sumaria), } 77 \text { (ingresos del trabajador } \\
\text { independientes). Después del análisis de "No } \\
\text { respuesta" y las depuraciones respectivas, la } \\
\text { muestra fue } 1442 \text { hogares de Piura. Para el caso } \\
\text { de los indicadores de desempeño financiero se } \\
\text { recurrió a la basa de datos de la SBS (2019). }\end{array}$ & $\begin{array}{l}\text { ENAHO (2019), fue } \\
\text { depurada en base a } \\
\text { confiabilidad estadística } \\
\text { y grado de "no } \\
\text { respuesta". Los datos de } \\
\text { las IMF son de SBS } \\
\text { (2019), fueron tratados } \\
\text { por componentes de } \\
\text { solvencia, sostenibilidad } \\
\text { y calidad de la cartera. } \\
\text { La aplicación del } \\
\text { análisis factorial resultó } \\
\text { ser el más asertivo para } \\
\text { cumplir el objetivo } \\
\text { planteado. }\end{array}$ \\
\hline
\end{tabular}




\begin{tabular}{|c|c|c|}
\hline $\begin{array}{c}\text { Variables del } \\
\text { Modelo }\end{array}$ & 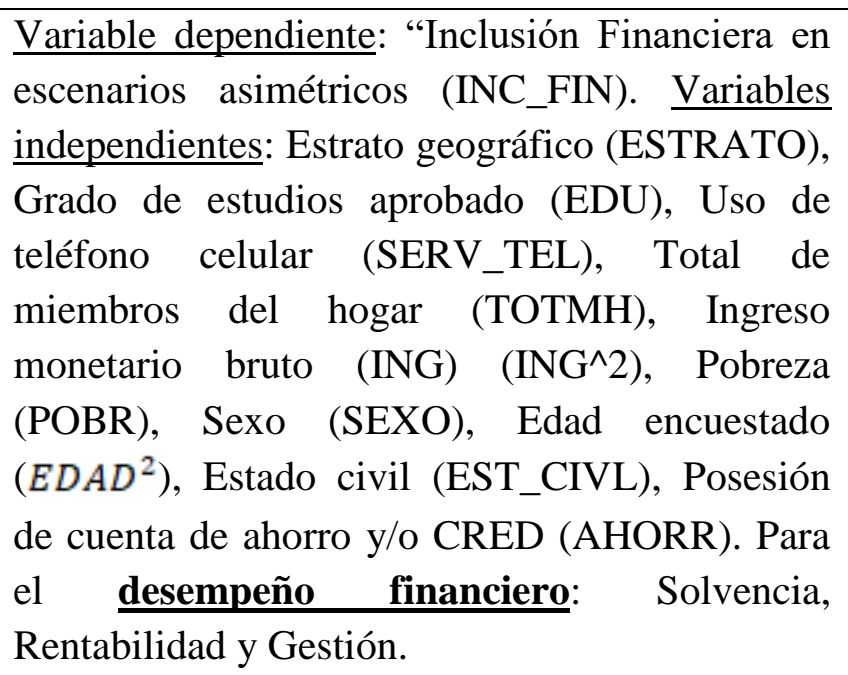 & $\begin{array}{lr}\text { Modelos logit y análisis } \\
\text { factorial reconocen } \\
\text { variables que son } \\
\text { detonantes } & \text { y/o } \\
\text { predictores } & \text { de } \\
\text { comportamientos que } \\
\text { permiten la } \\
\text { identificación de nuevas } \\
\text { hipótesis de trabajo que } \\
\text { se sustentan en fallos de } \\
\text { mercado (asimetría de } \\
\text { información) }\end{array}$ \\
\hline $\begin{array}{l}\text { Instrumentos } \\
\text { de } \\
\text { Investigación }\end{array}$ & $\begin{array}{l}\text { Para el análisis empírico de la investigación se } \\
\text { utilizó el modelo econométrico con variable } \\
\text { cualitativa- limitada específicamente el modelo } \\
\text { logit, el cual ha sido seleccionado por presentar } \\
\text { mejores indicadores econométricos y } \\
\text { contrastables con la evidencia empírica. }\end{array}$ & $\begin{array}{l}\text { Se utilizan los softwares } \\
\text { SPSS 20,0 y STATA } \\
15,0 \text { para integrarlos con } \\
\text { las bases de datos del } \\
\text { ENAHO y SBS. }\end{array}$ \\
\hline
\end{tabular}

